\title{
COMMENT
}

\section{Quantification of biomarker functionality predicts patient}

\section{outcomes}

\author{
Banafshé Larijani $\mathbb{D}^{1,2,3}$, James Miles ${ }^{1,2,3,4}$, Stephen G. Ward ${ }^{5}$ and Peter J. Parker ${ }^{6,7}$
}

Implementation of a quantitative molecular imaging method (iFRET), which determines receptor-ligand interactions, has led to the finding that patients with a low extent of PD-1/PD-L1 interaction in metastatic NSCLC, and malignant melanoma, display significantly worsened overall survival compared to those with a high level of interaction.

British Journal of Cancer (2021) 124:1618-1620; https://doi.org/10.1038/s41416-021-01291-3

\section{MAIN}

The hallmarks of cancer described by Hanahan and Weinberg illustrate the necessary conditions for the manifestation of malignant neoplastic diseases. ${ }^{1}$ One of these conditions is the evasion of immune-detection and avoidance of immune destruction. A mechanism by which cancers may evade immunesurveillance is dysregulation of inhibitory immune-checkpoints. Immune-checkpoints are comprised of inhibitory receptors, found on the cell surface of immune-cells, and cognate ligands, expressed on antigen presenting cells. The programmed death receptor 1 (PD-1) is an example of an immune-checkpoint receptor. When engaged with is complementary ligand, programmed death receptor ligand 1 (PD-L1), there is a reduction of immune activation. This is in part thought to be facilitated by the recruitment of the tyrosine phosphatases SHP-1 and SHP-2 to the ITSM of PD-1.2 Cancers may dysregulate this checkpoint by upregulating PD-L1, thus facilitating an evasion of immune surveillance and destruction by tumour-specific T lymphocytes.

Currently, monoclonal antibodies can be used to block PD-1/ PDL-1 interactions, thus restoring immune-mediated tumour detection and destruction. Whilst these therapies have shown promising therapeutic benefit, low response rates are encountered. In a recent study of NSCLC patients, those with a tumour proportion score of $50 \%$ or more exhibited increased response to pembrolizumab. However, only a $41 \%$ response rate was achieved in this cohort. ${ }^{3}$ To select patients who are likely to benefit from these treatments, a number of immunohistochemistry (IHC)-based assays are used to assess PD-L1 expression, in effect using ligand expression as a surrogate of checkpoint engagement. This indirect determination of checkpoint function has not proved effective in stratifying patients into treatment groups.

$A$ recent study assessed the efficacy of anti-PD-1/PD-L1 therapies in lung carcinoma, renal carcinoma and melanoma. In this study, patients' PD-L1 expression was assessed and patients were classified as PD-L1 positive or PD-L1 negative. Here, therapeutic benefit was seen in PD-L1 negative patients, implying that ligand expression is not a suitable biomarker to select patients for immune-checkpoint therapies. ${ }^{4}$ Hence, in order to overcome the shortcomings of IHC-based assays we developed a molecular imaging assay which quantifies PD-1/PD-L1 interaction states, alongside receptor and ligand expression (Fig. 1a). ${ }^{5}$ This assay is termed iFRET. Alternative assays exist which attempt to measure PD-1/PD-L1 interaction, however unlike iFRET these report on distances that likely reflect juxtaposition and cannot be relied upon to accurately report on checkpoint receptor engagement. For example, others have previously used an imaging algorithm which determines when PD $-1^{+}$and PD-L1 ${ }^{+}$cells are in close proximity $(\leq 20 \mu \mathrm{m}){ }^{6}$ However, these distances are greater than the diameter of a cell. By comparison, iFRET reports events at the $1-10 \mathrm{~nm}$ scale and can directly measure intercellular proteinprotein interactions.

The aforementioned iFRET assay was first compared with the Roche Ventana SP142 assay which is currently used to assess clinical PD-L1 expression. Crucially, the iFRET assay was able to detect a PD-1/PD-L1 interaction in 10 of the 11 PD-L1 negative ccRCC patients. ${ }^{5}$ This demonstrates that ligand expression is a poor surrogate of receptor engagement and that the direct functional PD-1/PD-L1 interaction needs to be quantified. Following this analysis, iFRET was used to assess PD-1/PD-L1 interaction states in 176 malignant melanoma patients with known clinical outcomes. One hundred and fifty-nine of these patients were assessed by IHC methods to determine their PD-L1 expression profiles. Of the 159 patients, 117 were PD-L1 negative and 42 were PD-L1 positive. In the PD-L1 negative group, 58 patients showed checkpoint interaction and 19 of 42 PD-L1 positive group showed no interaction state. ${ }^{5}$ This once again highlights the need for

\footnotetext{
${ }^{1}$ FASTBASE Solutions S.L., Kabi 612 Scientific and Technology Park of Bizkaia, Derio 48160, Spain; ${ }^{2}$ Cell Biophysics Laboratory, Ikerbasque, Basque Foundation for Science, Research Centre for Experimental Marine Biology and Biotechnology (PiE) \& Instituto Biofisikia (UPV/EHU, CSIC), University of the Basque Country, Leioa, Biscay, Spain; ${ }^{3}$ Cell Biophysics Laboratory, Department of Pharmacy and Pharmacology \& Department of Physics, Centre for Therapeutic Innovation, University of Bath, Bath, UK; ${ }^{4}$ Early Phase Trials and Sarcoma, Institut Bergonié, Bordeaux, France; ${ }^{5}$ Leukocyte Biology Laboratory, Centre for Therapeutic Innovation \& Department of Pharmacy and Pharmacology, University of Bath, Bath, UK; ${ }^{6}$ Protein Phosphorylation Laboratory, The Francis Crick Institute, London, UK and 'School of Cancer and Pharmaceutical Sciences, King's College London, London, UK

Correspondence: Banafshé Larijani (bl666@bath.ac.uk)

These authors contributed equally: Banafshé Larijani, James Miles
}

Received: 4 November 2020 Revised: 28 December 2020 Accepted: 26 January 2021

Published online: 15 March 2021 

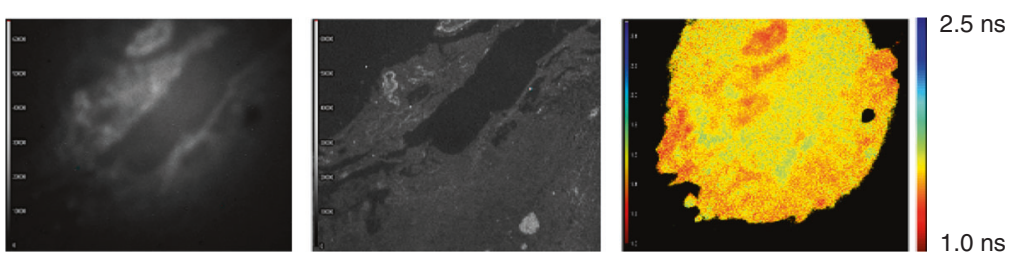

FRET efficiency $=24.12 \%$

iFRET quantifies PD-1/PD-L1 interaction state in non-small cell lung cancer.

In this sample, a FRET efficiency of $24.12 \%$ is detected, which indicates a PD-1/PD-L1 distance of $7.06 \mathrm{~nm}$.

b Survival vs PD-1/PD-L1 interaction

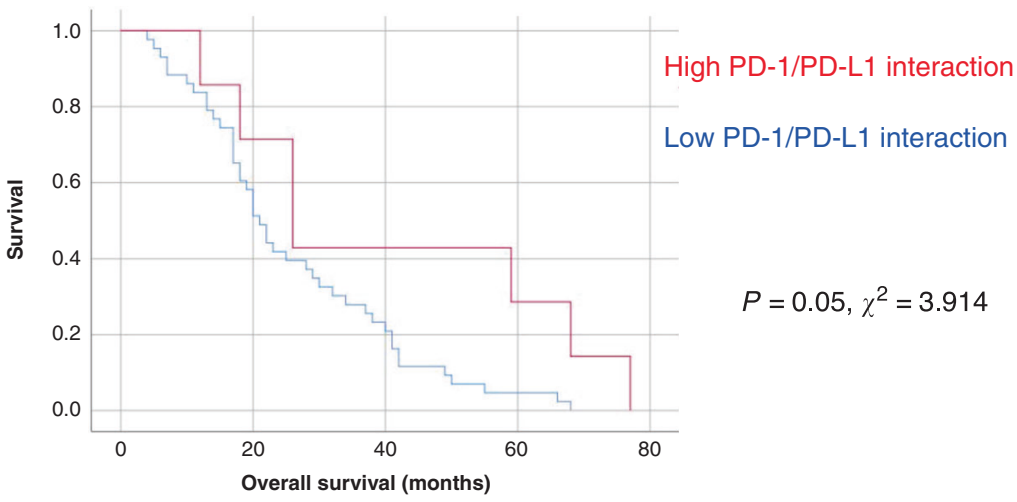

Survival vs PD-L1 intensity

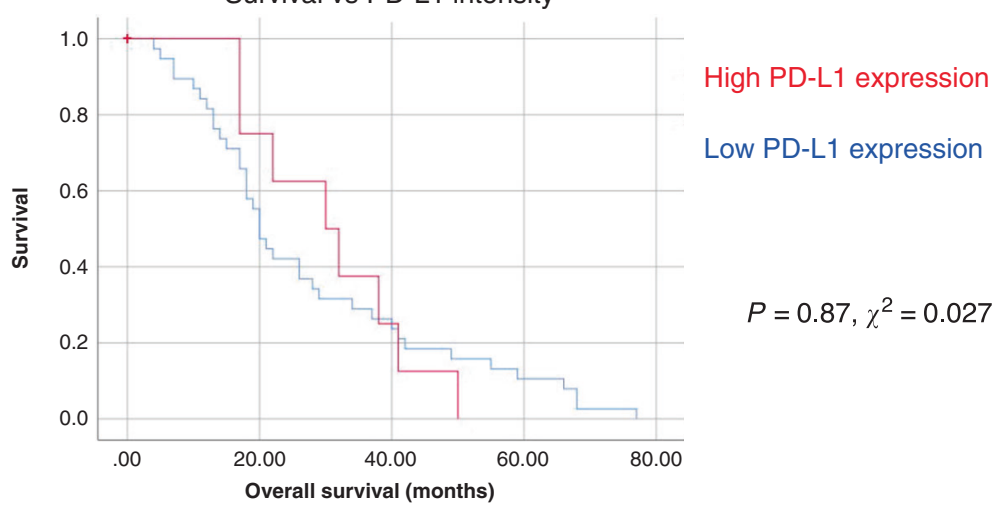

Fig. 1 iFRET quantifies PD-1/PD-L1 interaction state with patient outcome in malignant melanoma and NSCLC. a iFRET, a two-site assay simultaneously labels PD-1 and PD-L1 in patient samples. This allows the detection and quantification of PD-1 and PD-L1 interaction, alongside receptor and ligand expression. The black and white intensity maps show PD-1 and PD-L1 expression in an FFPE NSCLC sample. The pseudocolour lifetime map indicates FRET occurring between the labelled PD-1 receptor and PD-L1 ligand. This is correlated to a FRET efficiency of $20.77 \%$ and a receptor-ligand distance of $7.29 \mathrm{~nm}$. b iFRET correlates PD-1/PD-L1 interaction state with overall survival in malignant melanoma. (Top) Kaplan-Meier curve indicates that patients with a higher PD-1/PD-L1 interaction have a significantly worsened overall survival $\left(p=0.05, X^{2}=3.914\right)$. (Bottom) Kaplan-Meier curve correlates overall survival with PD-L1 expression. Here, PD-L1 expression does not correlate with patient outcome $\left(p=0.87, X^{2}=0.027\right)$. Modified from Sanchez-Magraner et al. ${ }^{5}$.

direct assessment of checkpoint interaction, and subsequent functionality, rather than indirect determination through the surrogate of ligand expression. Current methods are creating a scenario wherein patients may be enduring the side-effects of immune-checkpoint inhibition to gain no therapeutic benefit. Conversely, patients may miss these novel treatment opportunities which can potentially deliver increased survival rates.

As patient survival is often correlated to biomarker expression, a potential correlation between PD-1/PD-L1 and overall survival was assessed. We demonstrated firstly in melanoma that PD-L1 expression did not correlate to overall survival ( $p=0.87$ ) (Fig. 1b). Conversely, patient's PD-1/PD-L1 interaction state correlated with overall survival $(p=0.05)$. Here, those with a lower PD-1/PD-L1 interaction state experienced a significantly worsened overall survival (Fig. 1b). ${ }^{5}$ Following this, PD-1/PD-L1 interaction states were quantified in a cohort of anti-PD-1 treated metastatic NSCLC patients. As observed in the melanoma cohort, PD-L1 expression did not correlate with overall survival. In this NSCLC posttreatment cohort, those with a lower PD-1/PD-L1 interaction state again had a significantly worsened overall survival $(p=0.05) .^{5}$

Current literature suggests that an increase in PD-1/PD-L1 interaction would cause a decrease in overall survival due to immune-suppression. However, the aforementioned results suggest that in the cohorts examined, a higher interaction state may correlate with improved survival. It is surmised that a high PD-1/ PD-L1 interaction state infers tumour selection within patients, resulting in a subset of patients whose tumour facilitate immuneevasion via PD-1/PD-L1 interaction. It is this subset of patients who 
are predicted to respond to anti-PD-1/PD-L1 therapies and possibly should be considered for these treatments. Moreover, it remains unknown what extent of PD-1/PD-L1 disengagement is required to increase immune-mediated tumour destruction by tumour infiltrating lymphocytes.

As the pharmacodynamics of immune-checkpoint interactions are not monitored, it could be that only a partial or transient disengagement of PD-1/PD-L1 is required to observe an improved clinical outcome. It would be beneficial therefore to use iFRET to assess checkpoint interaction over time to observe how these checkpoint interactions evolve after treatment. It could be that the use of anti-PD-1 therapeutic antibodies results in a thresholding of T lymphocytes. In this instance, after treatment with anti-PD-1 therapies, a subset of $\mathrm{T}$ lymphocytes undergo reactivation and are polarised towards the tumour via recognition of tumour antigens. The increase of patient survival in those with a high interaction state may highlight that only a partial reactivation of $T$ lymphocytes is needed to re-mount an immune response.

Those patients with a low level of interaction and worsened survival may nevertheless benefit from alternative immune therapies. These tumours may evade the immune system by dysregulating CTLA-4/CD-80 or other immune-checkpoint interactions. Furthermore, no tumour will discretely dysregulate one pathway, in fact, a tumour may evolve to evade host immune response by modulating multiple pathways simultaneously, indicating a patient group who would benefit from dual checkpoint inhibitor therapies. ${ }^{7,8}$ In these instances, the functionality of multiple biomarkers should be quantified simultaneously in order to tailor patients to the most efficacious therapies.

The approach developed can be regarded as a companion diagnostic tool and can be utilised alongside routine IHC PD-L1 analysis using sections from the same fixed biopsies. In a wider context, this approach can be used to determine biomarker functionality to identify novel predictive biomarkers in a range of pathologies.

\section{ACKNOWLEDGEMENTS}

We thank Dr. Lissete Sánchez-Magraner and Christopher J Applebee (BSc, MSc) for their crucial work which has led into the results discussed in this paper. We thank Pierre Leboucher for the automation of the multiple frequency domain FLIM and Patel Poulam, clinical oncologist at Nottingham, for clinical discussions. We would also like to thank Audrey Colomba at the Francis Crick Institute for her help in PLA image acquisition.

\section{AUTHOR CONTRIBUTIONS}

B.L.-writing, review and editing. J.M.-writing, review and editing. S.G.W.- review and editing. P.J.P.—-review and editing

\section{ADDITIONAL INFORMATION \\ Ethics approval and consent to participate Not applicable.}

Consent to publish Not applicable.

Data availability The original data are with Cancer Research where the article is published.

Competing interests B.L. reports a patent for international (PCT) patent application no. PCT/EP2018/062719 "Kits, methods, and their uses for detecting cell-cell interactions". B.L. also reports the patent PCT/GB14/050715 "TSA amplification in
FRET/FLIM". As disclosed upon submission of the article for peer review, B.L. is a cofounder of FASTBASE Solutions. The competing interests are related to the interpretation of the clinical data. They have been and will be managed in the following manner: The iFRET platform was developed so that it could provide unbiased acquisition and interpretation of data. Hence, it was automated in a specific manner that the operator would not be making biased decisions. Moreover, all the patient samples are blinded during both acquisitions and interpretation of data. The parameters were revealed to us once we had completed the acquisition and interpretation of the data. P.J.P. reports being a co-founder and shareholder of FASTBASE Solutions S.L. outside the submitted work and has a patent for amplified FRET issued and licensed to FASTBASE Solutions and a patent for iFRET pending (FASTBASE license). The other authors declare no competing interests.

Funding information The original research was supported, in part, by Department of Education, Basque Government- IT1270-19, Elkartek grant (BG18) and the Spanish Ministry grant (MINECO) PROJECTS of EXCELLENCE (BFU2015-65625-P). P.J.P. was supported by a core grant to the Francis Crick Institute, from Cancer Research UK (FC001130), the UK Medical Research Council (FC001130) and the Wellcome Trust (FC001130).

Publisher's note Springer Nature remains neutral with regard to jurisdictional claims in published maps and institutional affiliations.

\section{REFERENCES}

1. Hanahan, D. \& Weinberg, R. A. Hallmarks of cancer: the next generation. Cell 144, 646-674 (2011)

2. Chemnitz, J. M., Parry, R. V., Nichols, K. E., June, C. H. \& Riley, J. L. SHP-1 and SHP-2 associate with immunoreceptor tyrosine-based switch motif of programmed death 1 upon primary human $\mathrm{T}$ cell stimulation, but only receptor ligation prevents T cell activation. J. Immunol. 173, 945-954 (2004).

3. Roach, C., Zhang, N., Corigliano, E., Jansson, M., Toland, G., Ponto, G. et al. Development of a companion diagnostic PD-L1 immunohistochemistry assay for pembrolizumab therapy in non-small-cell lung cancer. Appl. Immunohistochem. Mol. Morphol. 24, 392-397 (2016).

4. Nunes-Xavier, C. E., Angulo, J. C., Pulido, R. \& López, J. I. A critical insight into the clinical translation of PD-1/PD-L1 blockade therapy in clear cell renal cell carcinoma. Curr. Urol. Rep. 20, 1 (2019).

5. Sanchez-Magraner, L., Miles, J., Baker, C. L., Applebee, C. J., Lee, D. J., Elsheikh, S. et al. High PD-1/PD-L1 checkpoint interaction infers tumor selection and therapeutic sensitivity to anti-PD-1/PD-L1 treatment. Cancer Res. 80, 4244-4257 (2020).

6. Giraldo, N. A., Nguyen, P., Engle, E. L., Kaunitz, G. J., Cottrell, T.R., Berry, S. et al. Multidimensional, quantitative assessment of PD-1/PD-L1 expression in patients with Merkel cell carcinoma and association with response to pembrolizumab. J. Immunother. Cancer 6, 99 (2018).

7. Okazaki, T. \& Honjo, T. The PD-1-PD-L pathway in immunological tolerance. Trends Immunol. 27, 195-201 (2006).

8. Mahmoudi, M. \& Farokhzad, O. C. Cancer immunotherapy: wound-bound checkpoint blockade. Nat. Biomed. Eng. 1, 0031 (2017).

Open Access This article is licensed under a Creative Commons Attribution 4.0 International License, which permits use, sharing, adaptation, distribution and reproduction in any medium or format, as long as you give appropriate credit to the original author(s) and the source, provide a link to the Creative Commons license, and indicate if changes were made. The images or other third party material in this article are included in the article's Creative Commons license, unless indicated otherwise in a credit line to the material. If material is not included in the article's Creative Commons license and your intended use is not permitted by statutory regulation or exceeds the permitted use, you will need to obtain permission directly from the copyright holder. To view a copy of this license, visit http://creativecommons. org/licenses/by/4.0/.

(c) The Author(s) 2021 